\title{
Hipoglicemia na Infância: Resultados de um Protocolo de Avaliação Prospectiva em Crianças com Até 1 Ano de Idade
}

\begin{abstract}
RESUMO
O objetivo deste trabalho é relatar a experiência na aplicação de um protocolo de avaliação de hipoglicemia. Realizamos um estudo prospectivo com treze crianças com sintomas de hipoglicemia atendidas no Hospital de Clínicas de Porto Alegre, com idade média de 5,3 $\pm 4,5$ meses, das quais oito pacientes eram do sexo feminino. Os pacientes foram submetidos ao teste de jejum, estímulo com glucagon e dosagens de glicemia, lactato, $\mathrm{pH}$, ácidos graxos, insulina, peptídeo C, TSH, GH e cortisol, além de urina para avaliação de cetonúria. Dos treze pacientes avaliados, oito apresentaram hipoglicemia persistente e cinco apresentaram hipoglicemia transitória. O diagnóstico mais freqüente foi hiperinsulinismo persistente. Sugerimos a utilização de um protocolo simples de avaliação para hipoglicemia, que contempla as principais etiologias em crianças e que facilita o manejo desses pacientes. (Arq Bras Endocrinol Metab 2007;51/9:1493-1497)
\end{abstract}

Descritores: Hipoglicemia; Infância; Neonatos; Criança

\section{ABSTRACT}

Childhood Hypoglycemia: Results of Prospective Evaluation Protocols in Children with Up to 1 Year of Age.

The aim of this study is to present the experience of applying hypoglycemia evaluation protocol. We performed a prospective study with 13 children with hypoglycemia symptoms at the Hospital of Clinicas of Porto Alegre, with range age $5.3 \pm 4.5$ months and eight patients are female. The patients had been submitted to glucagon fasting test and blood glucose, lactate, $\mathrm{pH}, \mathrm{C}$ peptide, insulin, fatty acids, TSH, GH, cortisol, and urine ketones were measured. Eight patients presented persistent hypoglycemia and five presented transitory hypoglycemia. The most frequent diagnosis was persistent hyperinsulinism. We suggest the use of a simple protocol for the evaluation of hypoglycemia, which contemplates the identifications of the main etiologies in children and facilitates the handling of these patients. (Arq Bras Endocrinol Metab 2007;51/9:1493-1497)

Keywords: Hypoglycemia; Childhood; Neonates; Children

\footnotetext{
A HIPOGLICEMIA É UM ACHADO FREQÜENTE em recém-nascidos (RN), particularmente no primeiro ano de vida (1). Na maioria dos $\mathrm{RN}$ saudáveis, a freqüente observação dos níveis reduzidos de glicemia não está relacionada a qualquer problema clínico e reflete um processo normal de adaptação à vida extra-uterina (2). Aproximadamente $30 \%$ dos RN não conseguem manter seus níveis de glicemia maiores que $30 \mathrm{mg} / \mathrm{dl}$, caso sua primeira refeição seja atrasada em 3 a 6 horas após o seu nascimento (3). A hipoglicemia é geralmente causada por redução das reservas de energia, hiperinsulinemia e/ou excessivo gasto energético. $\mathrm{O}$ risco de hipoglicemia transitória está aumenta-
}

\section{artigo original}

\author{
TICIANA C. RODRIGUES \\ MAIKEL COLLI \\ Mauro A. Czepielewski
}

Serviço de Endocrinologia do Hospital de Clínicas de Porto Alegre e Programa de Pós-Graduação em Ciências Médicas: Endocrinologia, Universidade Federal do Rio Grande do Sul, Porto Alegre, RS.
Recebido em 14/05/07

Aceito em 18/06/07 
do em prematuros e pequenos para a idade gestacional (PIG), em filhos de mães diabéticas, em crianças com hiperinsulinismo, pacientes com sepse, hipotermia, anóxia perinatal e cardiopatia congênita $(4,5)$. Aproximadamente $50 \%$ desses episódios hipoglicêmicos são assintomáticos e detectados devido a algum fator predisponente associado (6). A maioria dessas crianças recuperase espontaneamente, entretanto 10 a $20 \%$ delas podem necessitar de infusão endovenosa de glicose (6). Uma distinção temporal é feita entre hipoglicemia transitória e persistente. Os episódios que durarem mais de uma semana são considerados persistentes e merecem uma investigação etiológica (7). As hipoglicemias recorrentes ou persistentes representam um grupo heterogêneo de distúrbios, com hipoglicemias mais severas e representando maior gravidade à criança.

A patologia mais comum nos quadros persistentes é o hiperinsulinismo, cujo diagnóstico é clínico e laboratorial, englobando um número conhecido e mesmo desconhecidos de mutações genéticas (8). Os casos severos e presentes no período neonatal são devido a mutações no gene do receptor tipo 1 da sulfoniluréia (SURI) ou do retificador interno dos canais de potássio da célula beta (kir 6.2) (9). Os casos leves são mais tardios e são, em alguns pacientes, devido a mutações nos genes da glucoquinase ou glutamato desidrogenase $(10,11)$.

A glicemia capilar, embora extremamente útil para a triagem de pacientes em risco de hipoglicemia, não estabelece o diagnóstico, havendo necessidade da realização da glicemia sérica. De um modo em geral, o ponto de corte utilizado para o diagnóstico de hipoglicemia é $40 \mathrm{mg} / \mathrm{dl}(12,13)$, mas há outros valores sugeridos conforme a faixa etária da criança; menores do que $20 \mathrm{mg} / \mathrm{dl}$ para prematuros e menores do que $30 \mathrm{mg} / \mathrm{dl}$ para os primeiros três dias de uma criança nascida a termo (14).

A hipoglicemia está associada a potenciais danos neurológicos e, assim, caracteriza uma situação clínica que exige diagnóstico preciso e manejo urgente. Apesar dessas peculiaridades, não há consenso sobre a melhor forma de abordagem, sendo propostos vários protocolos de investigação.
É importante salientar a extrema variabilidade do quadro clínico que pode associar-se à hipoglicemia. A tabela 1 descreve os sintomas mais freqüentes conforme a idade do paciente.

O objetivo deste trabalho é relatar a experiência do nosso serviço na aplicação de um protocolo de avaliação de hipoglicemia, organizado a partir de uma série de achados na literatura e implementado desde 2000.

\section{MÉTODOS}

Estudo prospectivo que incluiu 13 crianças com hipoglicemia acompanhadas pela equipe de endocrinologia do Hospital de Clínicas de Porto Alegre, no período de abril de 2001 a dezembro de 2004. Empregou-se, nesses pacientes, o mesmo protocolo de avaliação.

\section{Definição de hipoglicemia}

Foram incluídos pacientes que apresentavam glicemia capilar menor do que $40 \mathrm{mg} / \mathrm{dl}$ com confirmação por glicemia sérica.

\section{Protocolo para o teste de jejum}

Foram coletados exames durante a hipoglicemia espontânea. Nos pacientes que apresentavam apenas história de hipoglicemia ou que já estavam em tratamento com dieta ou infusão de glicose, procedeu-se ao teste de jejum para a indução do episódio hipoglicêmico, utilizando a idade do paciente como critério de tempo para ocorrência de hipoglicemia (15):

\begin{tabular}{lcccccc}
\hline Idade & $<$ meses & $6-8 \mathrm{~m}$ & $8-12 \mathrm{~m}$ & $1-2$ anos & $2-7$ anos & $>7$ anos \\
\hline Tempo máximo de jejum & $8 \mathrm{~h}$ & $12 \mathrm{~h}$ & $16 \mathrm{~h}$ & $18 \mathrm{~h}$ & $20 \mathrm{~h}$ & $24 \mathrm{~h}$ \\
\hline
\end{tabular}

\section{Protocolo de avaliação}

O teste foi iniciado pela manhã, mantendo-se o acesso venoso periférico heparinizado ou com soro fisiológico. Realizou-se glicemia capilar de hora em hora, ou antes, no caso de sintoma ou sinal suspeito. Quando a glicemia capilar atingisse valores menores que $40 \mathrm{mg} / \mathrm{dl}$, procedia-se à coleta de uma amostra de sangue para dosagens de glicemia (método enzimático colorimétrico-oxidase, $\mathrm{em} \mathrm{mg} / \mathrm{dl}$ ), lactato (método sinético-colorimétrico), $\mathrm{pH}$ (método de potenciometria), ácidos graxos (espectrometria de Massatandem), insulina (método de eletroquimioluminescência,

Tabela 1. Sinais e sintomas de hipoglicemia em diferentes estágios da criança.

\begin{tabular}{ll}
\hline Neonatos & Crianças \\
Sintomas autonômicos: & Sintomas autonômicos: \\
Palidez, sudorese e taquipnéia & Palidez, fraqueza, sudorese, náuseas, \\
& vômitos, dor abdominal e fome \\
Sintomas neuroglicopênicos: & Sintomas neuroglicopênicos: \\
Apnéia, hipotonia, irritabilidade, choro & Confusão, irritabilidade, cefaléia, distúrbio \\
anormal, dificuldade de sucção, convulsão & visual, mudança de comportamento, \\
e coma & convulsão e coma \\
\hline
\end{tabular}


em $\mathrm{mU} / \mathrm{ml}$ ), peptídeo $\mathrm{C}$ (método de eletroquimioluminescência, em $\mathrm{ng} / \mathrm{ml}$ ), tireotrofina (TSH) (método de eletroquimioluminescência, em $\mu \mathrm{Ui} / \mathrm{ml}$ ), hormônio de crescimento $(\mathrm{GH})$ (método quimioluminescência, em $\mathrm{ng} / \mathrm{dl}$ ) e cortisol (método eletroquimioluminescência, em $\mu \mathrm{g} / \mathrm{dl}$ ), além de urina para avaliação de cetonúria (método nitroprussiato de sódio). Após essa coleta, injetava-se glucagon na dose de $0,03 \mathrm{mg} / \mathrm{kg}$ intravenoso ou intramuscular (máximo de $1 \mathrm{mg}$ ), com posteriores coletas de glicemia nos tempos 20 e 30 minutos, cortisol e GH nos tempos 60, 90 e 120 minutos.

\section{Interpretação do teste}

A elevação da glicemia plasmática de pelo menos $25 \mathrm{mg} / \mathrm{dl}$ acima do valor inicial sugere fortemente o diagnóstico de hiperinsulinismo (16). No momento da hipoglicemia, níveis de insulina sérica maiores que $6 \mu \mathrm{U} / \mathrm{ml}$ e níveis de peptídeo $\mathrm{C}$ maiores que $0,6 \mathrm{ng} / \mathrm{ml}$ reforçam esse diagnóstico, associados a níveis reduzidos de cetonas e ácidos graxos, além de uma resposta normal ao cortisol e GH. Nas deficiências de cortisol e GH, as crianças apresentam-se cetóticas, os níveis de insulina estão reduzidos, o lactato é normal e não há resposta da glicemia ao estímulo com glugagon. Desordens da oxidação dos ácidos graxos são sugeridas por acidose metabólica, hipoglicemia cetótica e elevação dos níveis de ácidos graxos livres. Outros erros inatos do metabolismo são suspeitados quando há presença de acidose metabólica, distúrbios hidroeletrolíticos e alteração da função hepática. O protocolo recomenda que as amostras de sangue dos tempos 60, 90 e 120 minutos sejam armazenadas para posterior processamento, após o conhecido resultado das coletas basais, em função do elevado custo dessas dosagens laboratoriais.

\section{RESULTADOS}

Durante o período de utilização do protocolo, foram avaliadas 13 crianças com hipoglicemia. Dos 13 pacientes estudados, 5 pacientes eram do sexo feminino e 8 eram masculinos. A descrição dos pacientes com seus respectivos diagnósticos, sintomatologia e idade estão descritos na tabela 2 .

Os pacientes identificados pelos números $1,4,8$, 9 e 11 apresentaram hiperinsulinismo transitório em decorrência de suas patologias de base, não foram submetidos ao teste de jejum em função da gravidade de suas doenças. As seguintes orientações foram dadas: manter o aporte adequado de carboidratos, aguardar melhores condições clínicas e posteriormente, caso ainda fosse necessário, realizar o teste de jejum. Porém, todos apresentaram resolução das hipoglicemias com a recuperação clínica. Os pacientes restantes foram submetidos à avaliação. $\mathrm{O}$ protocolo foi efetivo em todos os pacientes estudados, sendo que nos pacientes 6 e 10 a avaliação do distúrbio genético contribuiu para a obtenção do diagnóstico. A descrição dos exames laboratoriais no basal e após os testes de estimulação estão descritos nas tabelas 3 e 4 , respectivamente.

Tabela 2. Descrição clínica e diagnóstico dos pacientes com hipoglicemia.

\begin{tabular}{|c|c|c|c|}
\hline Paciente & Idade & Sintomatologia & Diagnóstico \\
\hline 1 & $1 \mathrm{~h}$ de vida & PIG, retardo de crescimento intra-uterino, hipotonia & Baixo peso \\
\hline 2 & $1 \mathrm{~h}$ de vida & Crises convulsivas & Hiperinsulinismo persistente \\
\hline 3 & 3 dias & Crises convulsivas, irritabilidade & Hiperinsulinismo persistente \\
\hline 4 & 15 dias & PIG, apnéias, bradicardia, crise convulsiva & Baixo peso \\
\hline 5 & 4 meses & Crises convulsivas & Hiperinsulinismo persistente \\
\hline 6 & 6 meses & Hepatomegalia, retardo de desenvolvimento neuro-psicomotor & Galactosemia \\
\hline 7 & 3 meses & Hipotonia, choro anormal, hepatomegalia, icterícia & Panhipopituitarismo \\
\hline 8 & 5 dias & PIG, choro anormal, hipotonia & Prematuridade \\
\hline 9 & 20 dias & PIG, sepse neonatal, prematuro & Sepse neonatal \\
\hline 10 & 1 ano & Náuseas, vômitos, sudorese, icterícia, convulsão e coma & Glicogenose \\
\hline 11 & 17 dias & PIG, sepse neonatal, cianose peri-oral, hipotonia, convulsão & Prematuridade+sepse \\
\hline 12 & 1 ano & Cianose, hipotonia, torpor & Hiperinsulinismo persistente \\
\hline 13 & 4 meses & Vômitos, sudorese, tremores, choro anormal, hipotonia e icterícia & Deficiência de GH \\
\hline
\end{tabular}

Tabela 3. Resultados laboratoriais no momento da sintomatologia de hipoglicemia.

\begin{tabular}{cccccccc}
\hline Paciente & Glicemia & Cetonúria & pH & TSH & lactato & Insulina & peptídeo C \\
2 & 20 & negativo & 7,4 & 3,8 & 0,96 & 12,6 & 5,0 \\
3 & 36 & negativa & 7,4 & 2,9 & 0,85 & 8,9 & 2,6 \\
5 & 30 & negativa & 7,3 & 3,0 & 1,2 & 20,4 & 3,1 \\
6 & 35 & negativo & 7,1 & 3,9 & 1,0 & $<0,2$ & $<0,5$ \\
7 & 33 & positiva & 7,2 & 2,0 & 1,1 & $<0,2$ & $<0,5$ \\
10 & 17 & negativa & 7,0 & 3,5 & 5,06 & $<0,2$ & $<0,5$ \\
12 & 26 & negativa & 7,2 & 4,0 & 1,1 & 19 & 2,3 \\
13 & 20 & positiva & 7,3 & 2,5 & 0,8 & $<0,2$ & $<0,5$ \\
\hline
\end{tabular}


Tabela 4. Resultados basais ao teste de jejum com estímulo do glucagon em crianças com hipoglicemia.

\begin{tabular}{|c|c|c|c|c|c|c|c|c|c|c|c|}
\hline & $\begin{array}{c}\text { glicose } \\
\text { zero }\end{array}$ & $\begin{array}{c}\text { glicose } \\
20^{\prime}\end{array}$ & $\begin{array}{c}\text { glicose } \\
30^{\prime}\end{array}$ & $\begin{array}{c}\text { cortisol } \\
\text { zero }\end{array}$ & $\begin{array}{c}\text { cortisol } \\
60^{\prime}\end{array}$ & $\begin{array}{c}\text { cortisol } \\
90^{\prime}\end{array}$ & $\begin{array}{c}\text { cortisol } \\
120^{\prime}\end{array}$ & $\begin{array}{c}\mathrm{GH} \\
\text { zero }\end{array}$ & $\begin{array}{l}\mathrm{GH} \\
60^{\prime}\end{array}$ & $\begin{array}{l}\mathrm{GH} \\
90^{\prime}\end{array}$ & $\begin{array}{l}\mathrm{GH} \\
120\end{array}$ \\
\hline 2 & 20 & 55 & 68 & 14,8 & 13,7 & 15,0 & 33,0 & 1,21 & 3,0 & 7,0 & 10,0 \\
\hline 3 & 36 & 109 & 124 & 9,0 & 34,8 & 20,0 & 9,7 & 5,5 & 4,3 & 13,5 & 23 \\
\hline 5 & 30 & 94 & 134 & 23,0 & - & - & - & 2,0 & - & - & - \\
\hline 6 & 35 & 50 & 45 & 20,0 & 25,0 & 34,0 & 39,5 & 0,96 & 3,5 & 5,0 & 12,0 \\
\hline 7 & 33 & 40 & 45 & 2,5 & 5,0 & 5,5 & 8,0 & 0,55 & 2,30 & 1,80 & 3,00 \\
\hline 10 & 17 & 20 & 35 & 32,5 & 48,1 & 43,2 & 40,0 & 1,42 & 0,99 & 28,30 & 11,10 \\
\hline 12 & 26 & 55 & 70 & 21,1 & - & - & - & 13,9 & - & - & - \\
\hline 13 & 20 & 16 & 18 & 33,4 & 36,6 & 31,32 & 38,05 & 2,13 & 1,96 & 3,35 & 1,99 \\
\hline
\end{tabular}

\section{DISCUSSÃO}

Nosso trabalho analisa o emprego de um protocolo de avaliação de hipoglicemia em crianças menores de 1 ano de idade. Descrevemos como a simplificação e a objetivação da investigação à criança com hipoglicemia pode ser útil na prática clínica e eficiente na resolução diagnóstica.

Apesar de esse grupo de pacientes ser composto por uma amostra heterogênea, ele representa a freqüente sintomatologia observada e os diagnósticos mais encontrados, demonstrando a experiência de atendimento em um hospital terciário. Nos pacientes com hipoglicemia persistente, o diagnóstico mais freqüente foi hiperinsulinismo, o que é compatível com a descrição da literatura e representa uma secreção inadequada de insulina diante de níveis reduzidos de glicose, estando associado a níveis baixos de corpos cetônicos e ácidos graxos livres. No diagnóstico diferencial da hipoglicemia, o médico assistente deve levar em conta não apenas a glicose baixa, mas também o restante dos sinais e sintomas, assim como descrito na tabela 2. Um exemplo disso é a alteração hepática que favorece o diagnóstico diferencial entre sepse, hipopituitarismo, galactosemia, intolerância à frutose e doença do armazenamento do glicogênio (17).

O teste de jejum realizado em crianças é seguro, desde que realizado em ambiente hospitalar e com assistência médica adequada (15).

$\mathrm{Na}$ literatura brasileira, há descrição apenas de relatos de casos de pacientes com hipoglicemia ou revisões da literatura, sendo o nosso artigo o primeiro a descrever um grupo maior de pacientes com esse sintoma. Um outro estudo descreveu um protocolo semelhante de investigação, porém os níveis de peptídeo C e lactato não são coletados rotineiramente no momento basal e o tempo máximo de espera para hipoglicemia não é descrito. A dosagem de peptídeo C é realizada apenas em situações de suspeita de hipoglicemia factícia e os valores de glicemia e insulina para interpretação dos resultados também são distintos. Reforçamos a importância da dosagem do peptídeo C para reforço do diagnóstico de hiperinsulinismo e confirmação da origem endógena da insulina. Ainda nesse estudo, consideram-se os valores glicêmicos maiores que $40 \mathrm{mg} / \mathrm{dl}$ sobre o valor basal após teste de estímulo com o glucagon, enquanto no nosso protocolo consideramos os valores maiores que 25 $\mathrm{mg} / \mathrm{dl}$ sobre o valor basal, já que há comprovação de sensibilidade com esta resposta ao glucagon, não havendo a necessidade de um aumento de pelo menos $40 \mathrm{mg}$ na glicemia, em resposta ao mesmo teste. Utilizamos valores de insulina maiores que $6 \mu \mathrm{U} / \mathrm{ml}$ no momento da hipoglicemia, enquanto que na literatura os valores encontrados são diversos, variando desde $5 \mathrm{a} 10 \mu \mathrm{U} / \mathrm{ml}$ ou relação insulina/glicemia superior a $1: 4(6,18)$. Tal fato não compromete os resultados, visto que os nossos pacientes com hiperinsulinismo possuíam valores bastante elevados durante o episódio de hipoglicemia, inclusive níveis mais elevados que $10 \mu \mathrm{U} / \mathrm{ml}$.

Não realizamos as análises moleculares para $\mathrm{o}$ esclarecimento diagnóstico.

$\mathrm{Na}$ avaliação do diagnóstico diferencial de hiperinsulinismo responsivo ao diazoxido também devemos incluir a síndrome hiperinsulinismo-hiperamonemia, que está associada a níveis bastante elevados de amônia e que não possui a mesma base genética da desordem de secreção de insulina causada pelas mutações do gene da glutamato desidrogenase (19).

Felizmente, os nossos pacientes com hiperinsulinismo persistente responderam ao tratamento clínico com diazóxido e não necessitaram de intervenção cirúrgica. Um outro estudo com descrição de casuística internacional demonstrou que a maioria de seus pacientes respondeu de maneira satisfatória ao diazóxido, e que apenas $28,5 \%$ dos pacientes com hiperinsulinismo persistente necessitaram de intervenção cirúrgica (20). O tratamento de primeira escolha para o manejo do hiperinsulinismo da infância continua sendo o uso do diazóxido (21), porém este pode ou não resolver os episódios de hipoglicemia; nos casos não resolvidos, outras estratégias terapêuticas devem ser avaliadas, incluindo as ressecções pancreáticas. 
Em pacientes com fatores de risco conhecidos para hipoglicemia, deve-se reforçar a realização de glicemia capilar e não se deve realizar o teste de jejum, pois na maioria das vezes a hipoglicemia desaparece com a adequada alimentação e resolução da condição clínica de base. Entretanto, quando os níveis reduzidos de glicemia são prolongados e recorrentes, eles podem resultar em um efeito sistêmico agudo ou em alguma seqüela neurológica grave (22). Sendo assim, a abordagem diagnóstica deve ocorrer em paralelo com o tratamento da hipoglicemia. Nos casos com hipoglicemias graves e persistentes, sugerimos a utilização de um protocolo simples de avaliação para hipoglicemia, que contempla as principais etiologias em crianças e que desmistifica o manejo desses pacientes. Uma vez a etiologia definida, o tratamento adequado e dirigido pode ser empregado com sucesso.

\section{REFERÊNCIAS}

1. World Health Organization. Hypoglycaemia of the newborn Review of the literature. WHO/CHD/97.1. Available at: <http://www.who.int/reproductive-health/docs/hypoglycaemia_newborn.htm>. World Health Organization. Accessed in: January, 2007.

2. Cornblath $M$, Hawdon JN, Willians AF, Aynsley-Green $A$, Ward-Platt M, Schwartz R, et al. Controversies regarding definition of neonatal hypoglycemia: Suggested operational thresholds. Pediatrics 2000;105:1141-5.

3. Stanley CA, Baker L. The causes of neonatal hypoglycemia. $\mathbf{N}$ Engl J Med 1999;340:1200

4. Dalgiç N, Ergenekon E, Soysal S, Koç E, Atalay Y, Gücüyener $\mathrm{K}$. Transient neonatal hypoglycaemia long-term effects on neurodevelopmental outcome. J Pediatr Endocrinol Metab 2002;15:319-24.

5. Hawdon JM, Weddell A, Aynsley-Green A, Ward Platt MP. Hormonal and metabolic response to hypoglycaemia in small-for-gestational-age infants. Arch Dis Child 1993;68:269-73.

6. Damiani D, Dichtchekenian V, Setian N. Hipoglicemia na infância - ainda um desafio. J Pediatr 1997;73:231-8.

7. Brook D. Clinical Pediatric Endocrinology. Blackwell Science, 2001.

8. Matthew P, Young J. Persistent neonatal hyperinsulinism. Clin Paediatr 1988;27:148-51.

9. Bryan J, Aguilar-Bryan L. The ABCs of ATP-sensitive potassium channels: more pieces of the puzzle. Curr Opin Cell Biol 1997;9:553-9.
10. Glaser B, Kesavan P, Heyman M, Davis E, Cuesta A, Buchs A, et al. Familial hyperinsulinism caused by an activating glucokinase mutation. N Engl J Med 1998;279:340-5.

11. Stanley CA, Fang J, Kutyna K, Hsu BY, Ming JE, Glaser B, et al. Molecular basis and characterization of the hyperinsulinism/hypermmonemia syndrome; predominance of mutations in exons 11 and 12 of the glutamate dehydrogenase gene. HI/HA Contributing Investigators. Diabetes 2000;49:667-73.

12. Srinivasan G, Pildes RS, Cattamanchi G. Plasma glucose values in normal neonates: A new look. J Pediatr 1986;109:114.

13. Yamauchi Y. Hypoglycemia in healthy, full-term breast-fed neonates during the early days of live: Preliminary observation. Acta Paediatr Jpn 1997;39:44.

14. Cornblath M, Reisner SH. Blood glucose in the neonate and its clinical significance. N Engl J Med 1965;273:378.

15. Morris AAM, Thekekara A, Wilks Z, Clayton PT, Leonard JV, Aynsley-Green A. Evaluation of fast for investigating hypoglycaemia or suspected metabolic disease. Arch Dis Child 1996;75:115-9.

16. Lteif AN, Schwenk WF. Hypoglycemia in infants and children. Endocrinol Metab Clin North Am 1999;28:619-45.

17. Lee R, Choo-Kang Chen-Chih JS, Counts DR. Cholestasis and Hypoglycemia: Manifestations of congenital anterior hypopituitarism. J Clin Endocrinol Metab 1996;81:2786-9.

18. Stanley CA. Advances in diagnosis and treatment of hyperinsulinism in infants and children. J Clin Endocrinol Metab 2002;87:4855-9.

19. Stanley CA, Lieu YK, Hsu BY, Burlina AB, Greenberg CR, Hopwood $\mathrm{NJ}$, et al. Hyperinsulinism and Hyperammonemia in Infants with Regulatory Mutations of the Glutamate Dehydrogenase Gene. N Engl J Med 1998;338:1352-7.

20. Ismail D, Werther G. Persistent hyperinsulinism hypoglycaemia of infancy: 15 years' experience at the Royal Children's Hospital. J Pediatr Endocrinol Metab 2005; 18:1103-9.

21. Hussain K. Ketotic hypoglycaemia in children with diazoxide responsive hyperinsulinism of infancy. Eur J Pediatr 2005; 164:387-90.

22. Fluge G. Neurological findings at follow-up in neonatal hypoglycaemia. Acta Paediatr Scand 1975;64:629-34.

Endereço para correspondência:

Ticiana C. Rodrigues

Serviço de Endocrinologia

Hospital de Clínicas de Porto Alegre

Rua Ramiro Barcelos 2350, Prédio 12, 4 andar

90035-003 Porto Alegre, RS

Fax: (51) 2101-8777

E-mail: tcostarodrigues@terra.com.br 